\title{
Volumetric and Space Requirements of the Offshore Workforce: The Effects of Donning a Survival Suit.
}

\author{
Robert J. LEDINGHAM, Arthur D. STEWART \\ Institute of Health and Welfare Research, Robert Gordon University, Aberdeen, UK
}

\begin{abstract}
http://dx.doi.org/10.15221/13.317

Confined space working is common place within the offshore oil and gas infrastructure and it is a person's absolute size that governs his/her fit within this built environment. The design and adjustability of the environment must be based on the assumed size of the workforce, most commonly assumed to be the $95^{\text {th }}$ percentile of the male size. The last anthropometric survey of the UK offshore workforce was conducted almost 30 years ago and since then the average weight of the workforce has increased by $19 \%$, although the size and shape change associated with this increase remains unknown. With advances in portable 3D scanning technology and its potential for anthropometric measurement, this study aimed to quantify the volumetric and space requirements of the offshore workforce and size increases associated with donning personal protective equipment. Forty-three male participants were measured using both a static Hamamatsu and a portable Artec L 3D scanner in three different clothing assemblages. Volumetric and linear measures indicated a $71.3 \%$ increase in total body volume and a $101.9 \%$ gain in space requirements associated with donning a survival suit. Size increases due to survival clothing was found to have a close relationship with BMI; smaller individuals increase in body volume and space requirements comparatively more than their larger counterparts, $r=0.815$ and $r=0.659$ respectively. This pilot study identifies a need for further research into space requirements, especially in confined spaces and using specialist clothing.
\end{abstract}

Key words: 3D scanning, anthropometry, body volume, space requirements, survival clothing

\section{Introduction}

Offshore oil and gas workers are routinely required to live, work and travel in confined conditions. Space limitation leads to greater physiological stress, and adversely affects workers' safety and efficiency within these environments [1]. The current UK offshore workforce is now $19 \%$ heavier than their counterparts in the mid-1980's although their precise size and shape is unknown. Traditional anthropometry, as used in the original sizing survey [2], provides limited information about human body shape and can prove time consuming and costly in large population studies. Thus it is necessary to re-assess the actual size and shape of the workforce, to determine present day space requirements.

Although recent population surveys are available, the offshore environment appears to recruit individuals of atypical physique compared to that of the general population [3]. Furthermore, knowledge of the form-fitting body shape is not necessarily enough to understand. There is a need for body size to be measured in everyday clothing, as well as when wearing personal protective equipment, which can increase an individual's space requirement, alter ergonomic fit, and under some circumstances critically influence emergency escape. 3D scanning in large scale anthropometric population surveys has become common practice, recently used in the CAESAR [4] and SizeUK [5] surveys. While both studies were comprehensive they remained time consuming, costly and labour intensive, and relied on fixed, laboratory based scanners. However, advances in 3D portable scanning technology opens a whole new realm of measuring, without requiring participants to attend laboratory facilities. The present study is part of a larger project which will measure the offshore workforce at their place of work. This will increase the speed and selectivity of data collection, as well as measure body shape and quantify the size effect specialist survival clothing has on space requirements.

\section{Method}

Participants provided informed consent to participate in the study which received institutional ethics approval. Forty-three healthy males aged $31.2 \pm 12.0$ y attended the 3D scanning facility for all scans acquired during a single session. All individuals were screened pre-measurement for photosensitive epilepsy due to the strobe flash of the Artec $L$ camera. Each subject was measured with a Hamamatsu BLS9036 3D scanner (Hamamatsu Photonics, Japan) and an Artec L portable 3D 
scanner (Artec Group, Luxembourg) standing upright with arms by the sides ('egress position') in form-fitting, regular and the survival clothing (500 series helicopter passenger immersion suit; Survivtec Group, Birkenhead, UK) as illustrated in figure 1.

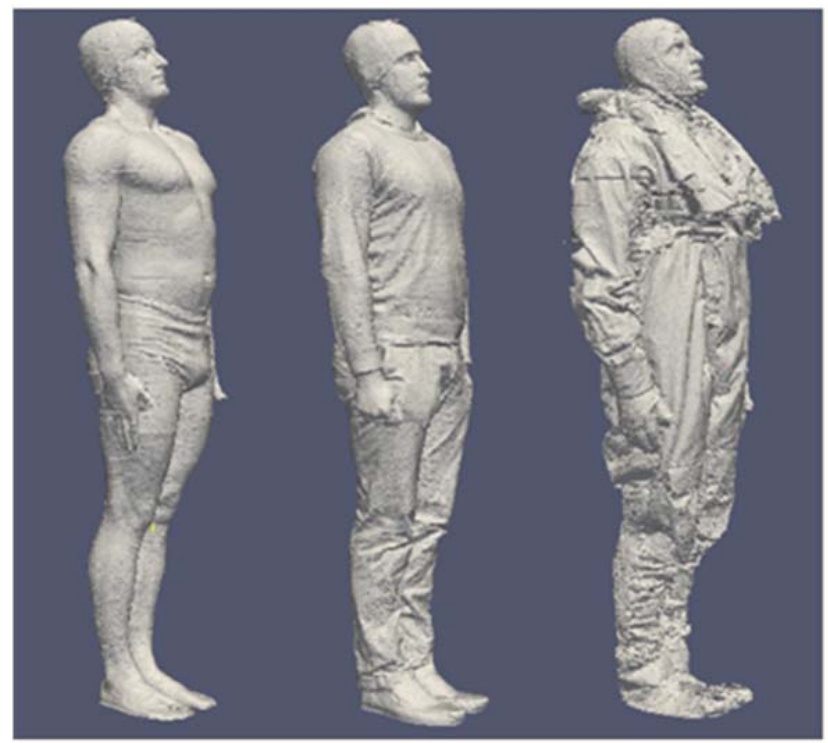

Figure 1. The three clothing assemblages in the 'Egress Position'.

Each scan was repeated, and all 12 scans per participant were completed within a single measurement session lasting less than one hour. Each scanner's dedicated software was used to extract all linear and volumetric measurements. The space footprint was calculated as the maximum area defined by bi-deltoid shoulder breadth and chest depth in a transverse plane as illustrated in figure 2. Participants were asked to stand in the egress position to minimise their space footprint and simulate the position they would be expected to adopt in an emergency muster station. Absolute volumes for different clothing assemblages were calculated along with commensurate increases in space footprint. Mean values from the duplicate scans were used in all calculations. Delineation of the arm-torso and leg-torso planes enabled the segmentation of the body and thus calculation of abdominal volume [6].

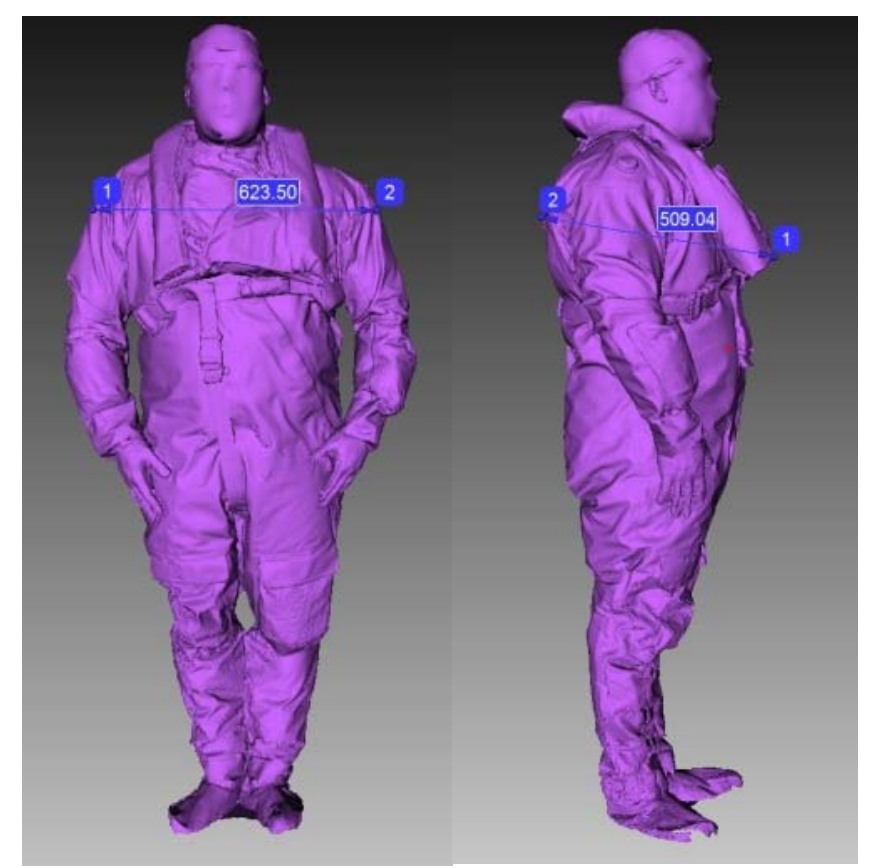

Figure 2. Bi-deltoid breadth and A-P chest depth measurements using the linear caliper in Artec Studio 9 software. 


\section{Results}

Table 1. Physical characteristics of participants.

\begin{tabular}{cc} 
Age $(\mathrm{y})$ & $31.2 \pm 12.0$ \\
Weight $(\mathrm{kg})$ & $84.1 \pm$ \\
Height $(\mathrm{cm})$ & $178.8 \pm 7.5$ \\
$\mathrm{BMI}\left(\mathrm{kg} \cdot \mathrm{m}^{2}\right)$ & $26.2 \pm 4.3$ \\
Abdom vol / vol (\%) & $46.4 \pm 10.3$ \\
\hline
\end{tabular}

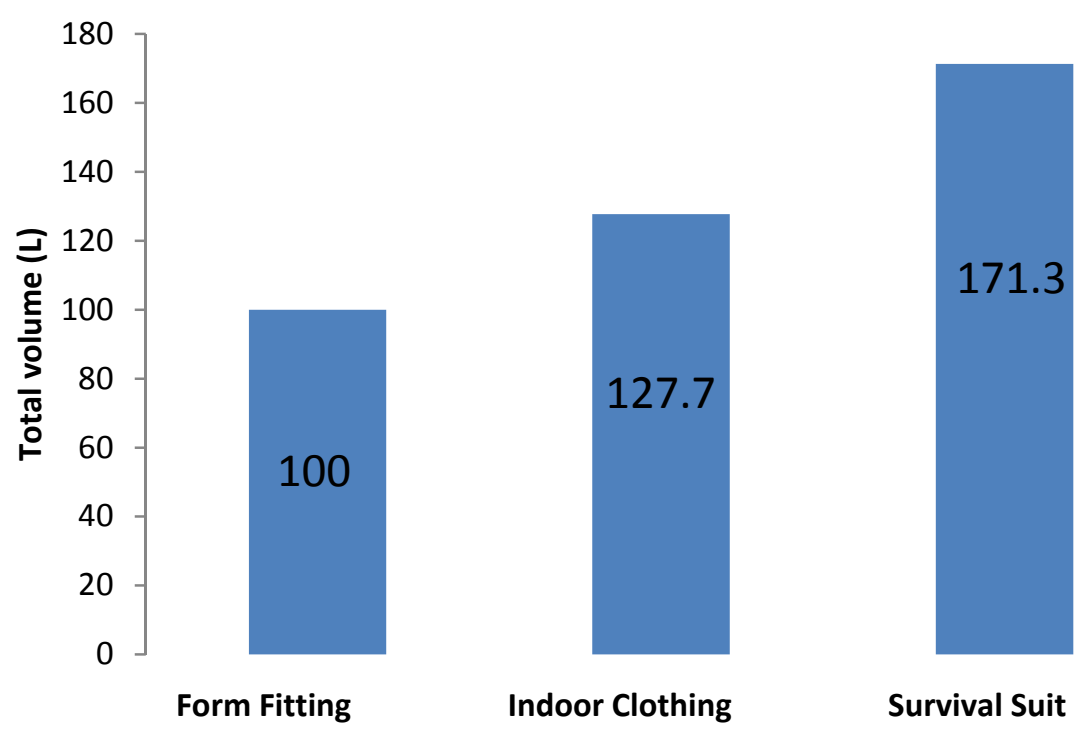

Figure 3. Volumetric increases related to clothing assemblages.

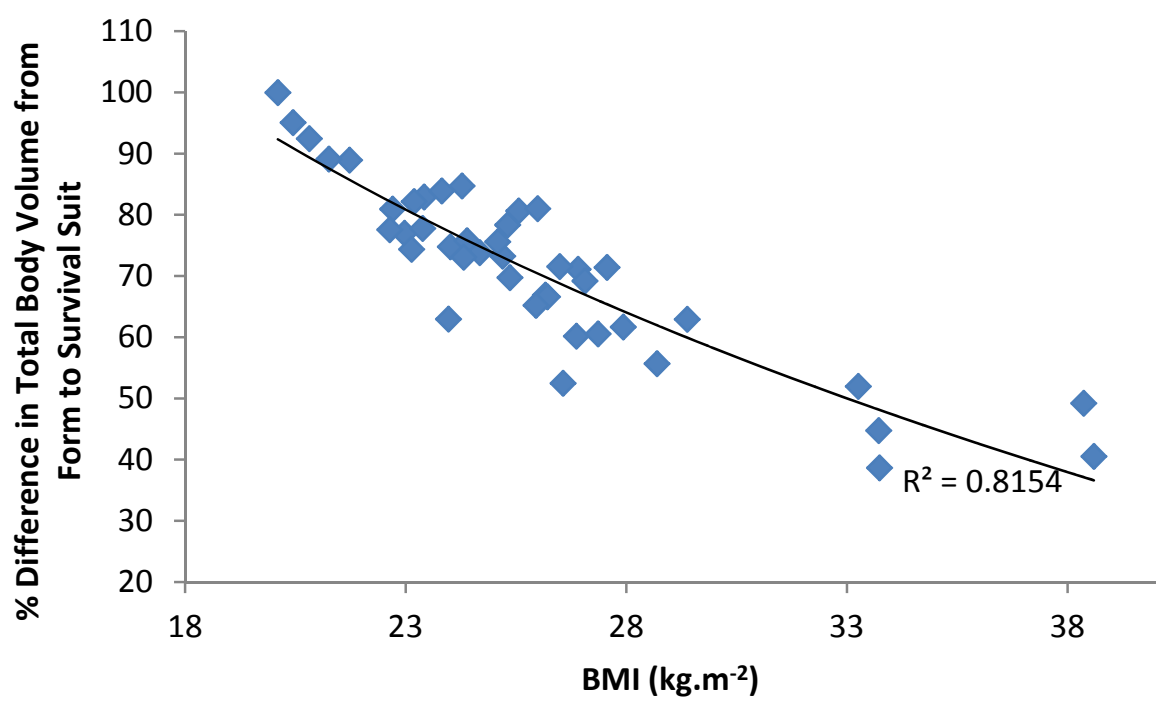

Figure 4. The effect of BMI on total body volume increase between form/survival suit. 


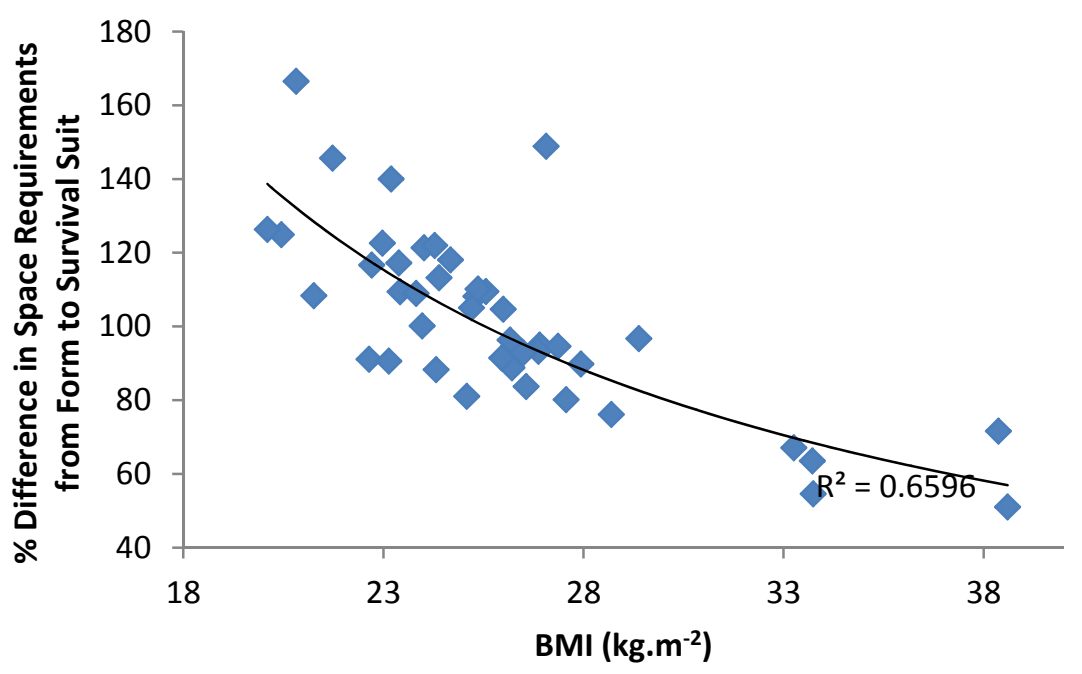

Figure 5. The effect of BMI on space requirement increase between form/survival suit.

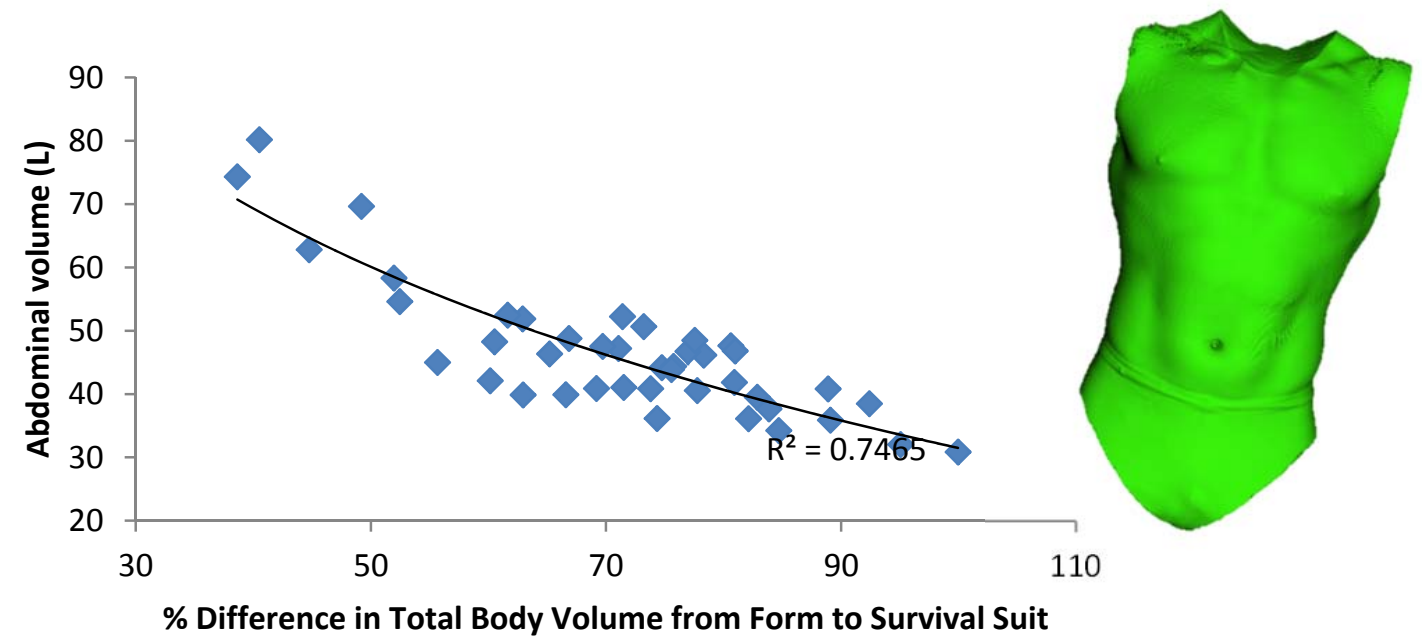

Figure 6. The effect of abdominal volume on \% difference in total body volume from form fitting clothing to survival suit.
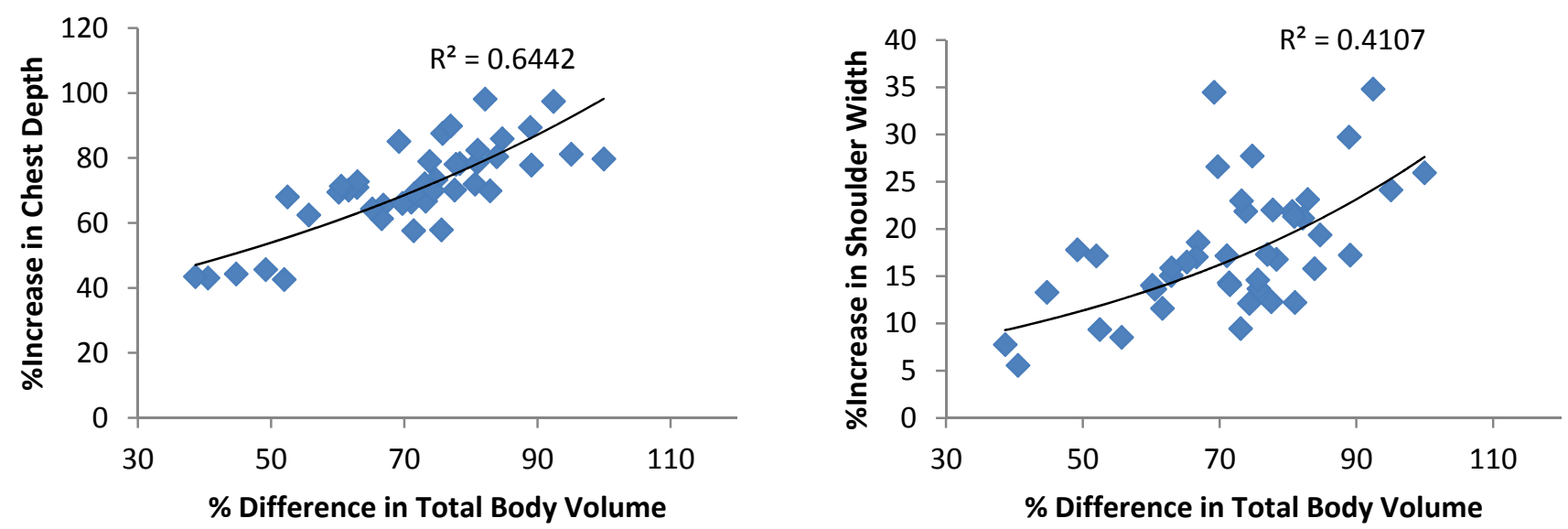

Figure 7. \% increase in direct measures against \% increase in total body volume. 


\section{Discussion}

These initial findings show that different clothing assemblages have a dramatic effect on the minimum space requirements of an individual, with an average increase in volume between form fitting clothing and a survival suit of $71.3 \%$ (figure 3 ), which corresponds to an increased space footprint of $101.9 \%$ represented in figure 8.

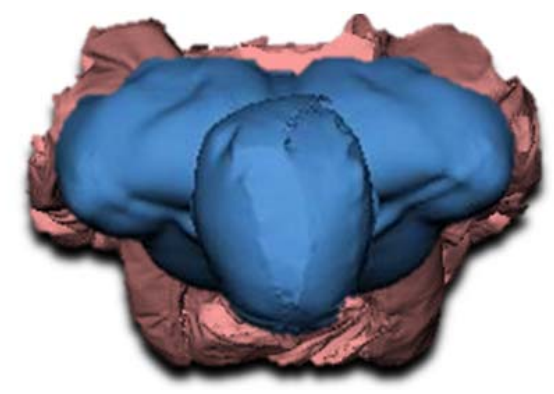

Figure 8. Increase in space requirements when wearing a survival suit and re-breather.

This dramatic increase in space requirements due to clothing assemblage raises important implications for the ergonomic design of the offshore working environment. As current infrastructure design is informed by data 30 years old since which time the UK prevalence of obesity has trebled, it is unlikely to represent the current workforce. Furthermore, body dimensions in extant data have not to date taken account of the size of personal protective equipment worn in today's offshore environment. With recent emergency situations in the UK offshore sector, particularly involving passenger helicopters, there has been an industry-wide focus on safety which has included a drive for understanding the role of space availability within aircraft to enable ease of egress during emergencies. Recognising space limitation, the International Marine Organisation propose changes to their Life Saving Code standards in relation to lifeboat capacities; increasing maximal space requirements for individuals from $430 \mathrm{~mm}$ to $575 \mathrm{~mm}$ ( $95^{\text {th }}$ percentile of bi-deltoid breadth), decreasing lifeboat capacities by $\sim 33 \%$ [7].

When donning a survival suit the relationship between BMI increase and total body volume/space requirement increases was shown to be negative, showing that smaller individuals increase in total body volume $(r=0.815)$ and space requirements $(r=0.659)$ proportionally more than their larger counterparts. Greater volume and space requirement increases in smaller individuals may be due to poor fit of the suit, leaving extra material and capacity for trapping air. The poorer fit in smaller individuals is primarily seen across the chest $(r=0.644)$ and abdominal area $(r=0.747)$ rather than across the shoulders which shows no relationship with proportional increases in total body volume $(r=$ 0.411). The 'one size fits all' re-breather adopted in the UK offshore industry may be attributed to a small proportion of this poor fit across the chest and abdomen in smaller individuals. Although overall poor fit leading to excess material bunching and trapping air appears to be the major contributing factor to the proportionally greater increases in total body volume seen in smaller individuals once donning a survival suit. Excess suit material folds in smaller individuals might represent a snagging hazard in emergency escape situations.

An important implication of this poor fit is additional trapped air in the survival suits. Although a survival suits thermal insulation in the event of cold water immersion is primarily provided by trapped air, the additional buoyancy this confers will impede an individual's ability to escape from a submerged helicopter. The additional buoyancy hinders escape as the forces it applies on the body need to be overcome for the occupant to pull him/herself down from the roof, or floor if helicopter has capsized, to reach the emergency exit to escape. As this additional buoyancy is also fluid, a major concern is that the trapped air migrates down to the feet of the survival suit making it difficult for the wearer to right themselves while in the water [8].

The aforementioned issues with survival suit sizing, confined space requirements and the knowledge that the original sizing survey data are now out of date has led for an industry initiative for current anthropometric size and shape data to be acquired. The current study forms essential preparatory research for this initiative, which will quantify the size and shape of the UK offshore workforce. The resulting data will inform a range of applications including infrastructure design, personal protective equipment, standard operating procedures, confined space working practice, lifeboat/helicopter loading capacities and any other space limited environment found offshore. 

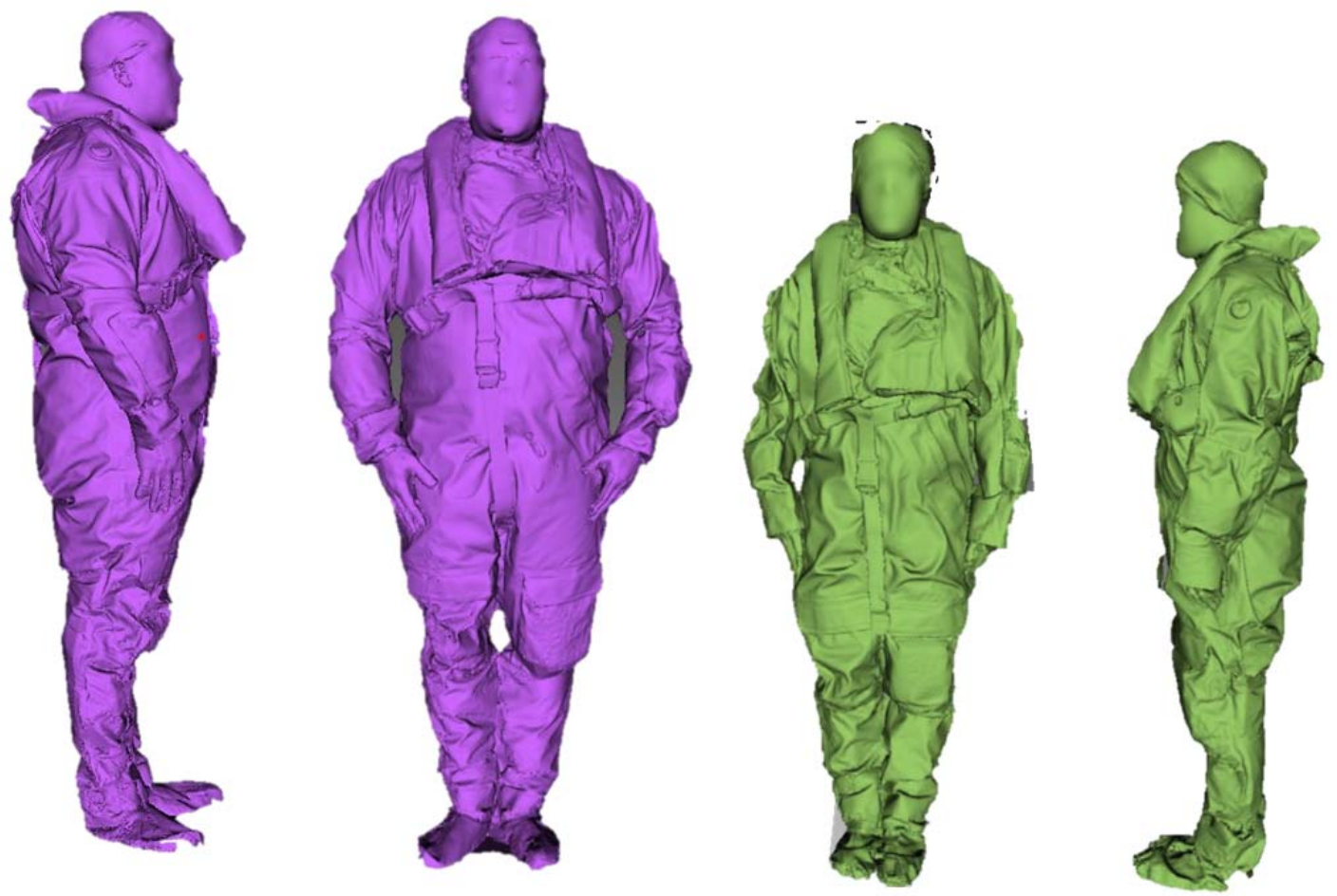

Figure 9. Illustrating the difference in snugness of fit between a large and small individual.

\section{Conclusion}

Size increases as a result of donning a survival suit, but proportionately more so in smaller individuals with potentially important implications for safety. As the mean body size of the global population increases, further research is warranted in space requirements, especially in confined spaces and using specialist clothing.

\section{Acknowledgements}

Oil and Gas UK, the UK offshore industry representative body responsible for health and safety, along with the UK government's Technology Strategy Board provided funding to complete this initial pilot study along with a large scale 3D scanning survey of the UK offshore workforce. The survey will be completed by researchers from Robert Gordon University.

\section{References}

1. Lowe BD et al (2001). Electromyographic and discomfort analysis of confined-space shipyard welding processes. Applied Ergonomics 32, 255-269.

2. Light, I.M. \& Gibson, M. (1986). Percentage body fat and prevalence of obesity in a UK offshore population. British Journal of Nutrition 56, 97-104.

3. Hsiao, H., Long, D. and Snyder, K., 2002. Anthropometric differences among occupational groups. Ergonomics. 45:2, 136-152.

4. Robinette, K. M. et al., 2002. Civilian American and European surface anthropometry resource (CAESAR) final report, volume 1: Summary. Interim report 1997-2002. Warrendale, PA: SAE International.

5. Wells, J.C.K., Treleaven, P. \& Cole, T.J. (2007). BMI compared with 3-dimensional body shape: The UK national sizing survey. American Journal of Clinical Nutrition, 85, 419-425.

6. McConville, J.T. \& Churchill, T.D., 1980. Anthropometric Relationships of Body and Body Segment Moments of Inertia.

7. Kozey, J. et al., 2005. The impact on immersion suit use on lifeboat capacities. resented:36th Annual Conference of the Association of Canadian Ergonomists.

8. Coleshaw, S. R. K., 2006. Investigation of removable exits and windows for helicopter simulators. Aberdeen: OPITO. 